\title{
A Preliminary Discussion of the ACATECH 4.0 and AHP to Measure Enterprise Maturity Level Index
}

\author{
Tainá da ROCHA, Anderson Luis SZEJKA ${ }^{1}$, Osiris CANCIGLIERI JUNIOR and \\ Eduardo de Freitas ROCHA LOURES \\ Industrial and Systems Engineering Graduate Program, Pontifical Catholic University \\ of Parana, Curitiba, Brazil
}

\begin{abstract}
Many organizations have redesigned their measurement systems to ensure that they reflect their current environment and strategies. Thus, it is extremely important that the responsible manager knows all the strengths and weaknesses of his organization, having all the maturity axes mapped, highlighting his strengths and weaknesses, to anticipate problems, becoming a company with greater potential competitiveness, because the failure is not to ignore the problem, but to ignore it. Given this, when measuring the Maturity Level Index, you can get an overview of the organization, becoming a radar to know the strengths and weaknesses, thus providing a basis for formulating a decision making and strategy to implement actions to improve performance and organizational maturity. The Acatech Industrie 4.0 (AI4MI) + AHP maturity index has the principle of providing companies with a guide for this transformation, based on the assessment of weaknesses or disagreements with the objective in the action plans, thus obtaining a continuous improvement in the evaluated stages, generating knowledge from the data, to transform the company into an agile organization, with quick decision making and adaptation in multiple business scenarios and different areas of the company. This article presents a preliminary discussion on the benefits of this proposed model for analyzing the measurement of the ACATECH + AHP Maturity Level Index, as to its advantages, results, added value.
\end{abstract}

Keywords. Maturity Index, Industry 4.0, Road Map, Decision Support, Performance Measurements, Operations Strategy.

\section{Introduction}

Currently, what governs the rules of competitiveness and survival of a company are: technological innovation, creativity, quality, low cost, customer satisfaction. And correlating all these factors is not an easy task. In recent times, much has been said about Industry 4.0 and its benefits and impacts on industry and society. The 4th Industrial Revolution brings us topics such as the Internet of Things (IoT), digitalization, intelligent and independent processes, big data, cloud storage, and many other macro themes [1]. The fact is that all this transformation causes curiosity, but also fears about culture, the way of thinking, and new ways of manufacturing. Industry 4.0 is not only about

\footnotetext{
${ }^{1}$ Correspondig Author, Mail: anderson.szejka@pucpr.br.
} 
connectivity between machines, processes, and products, but also about growth and organizational development in general [2].

The use of new technologies and the implementation of new knowledge through new information processing metrics inevitably brings us new ways of working, new jobs with which we need professional knowledge and experience [3]. Therefore, the first challenge and task to be carried out before any implementation tool presented by Industry 4.0 is to prepare the environment and culture of the whole society that will be directly and indirectly involved with this type of industrial revolution, thus designing a strategy for implementation aligned with your business strategy $[1 ; 4]$.

The concern with meeting the client's personalization requirements at the desired level of quality, competitive selling price, low manufacturing cost and profitability to remain competitive in the market is a complex and exhausting task [5]. For its implementation, a previous study must be carried out to map the environment to be modified, type of product, mission, and vision of the company and, thus, be able to be more assertive when performing the step by step for the application of maturity index measurement. Companies will only be able to enjoy the benefits of applying this tool if they implement the steps correctly and respecting all their stages in the process.

Performance management is a key factor in identifying skill gaps in any area of an organization or individual. Thus, it is of great importance to link the company's goals, even employees, to the company's Industry 4.0 strategy, [1]. The question is which is the best method, and how to interpret the results obtained.

This article will be structured as follows: Session 2 is dedicated to the problem statement; Section 3 presents the background, example work to promote the discussion and presents the highlighted model; Session 4 deals with the preliminary discussion between the advantages and disadvantages on the presented methods and, finally, session 5 presents the conclusion.

\section{Problem Statement}

Competitiveness in the market, customer requirements, rapid adaptation to changes, connectivity, and digitalization of data bring us to the reality of change. The question is: are our factories ready to receive this transformation? What is the impact of measurement systems on the organization's competitiveness and strategy? What method to measure maturity index can organizations use to ensure that their systems evolve, contributing to the increase in maturity? The fact is that many managers see the need for change, both physical and cultural, but it is difficult to measure the distance between the current state of reality and the desired state to be achieved. For such questions to be answered, it is necessary to know the level of maturity existing in the organization, and for that it must be clearly known which method to use, and most importantly, correctly interpret the extracted data so that action plans and strategies can be executed. However, knowing how to apply and interpret the best method is not a simple action. 


\section{Contextualization}

Industry 4.0 brought with it facilities, autonomy, connectivity, integration between the real world and the virtual. But all these advantages are not available so soon, it is necessary to promote adaptations throughout the organization. This implementation is a highly complex transformation, which does not occur quickly, requiring analysis, modifications, investments not only in strategic and technological aspects, but also in social aspects.

ACATECH + AHP assists, from the company's business strategy, identifying strengths and weaknesses in various perimeters, assisting in the formation of simulated scenarios according to the result obtained, through the functional areas of Development, Production, Logistics, Services, Marketing and sales. Each functional area of this is classified into four sub-levels: Culture, Organizational Structure, Resources, and Information Systems. Each sub-level is classified into 6 classes that refer to the requirements of the implementation phases of the Industry 4.0 stages [6], namely: Adaptability, Predictive Capacity, Digitization, Computerization, Transparency, Visibility (Figure 1).

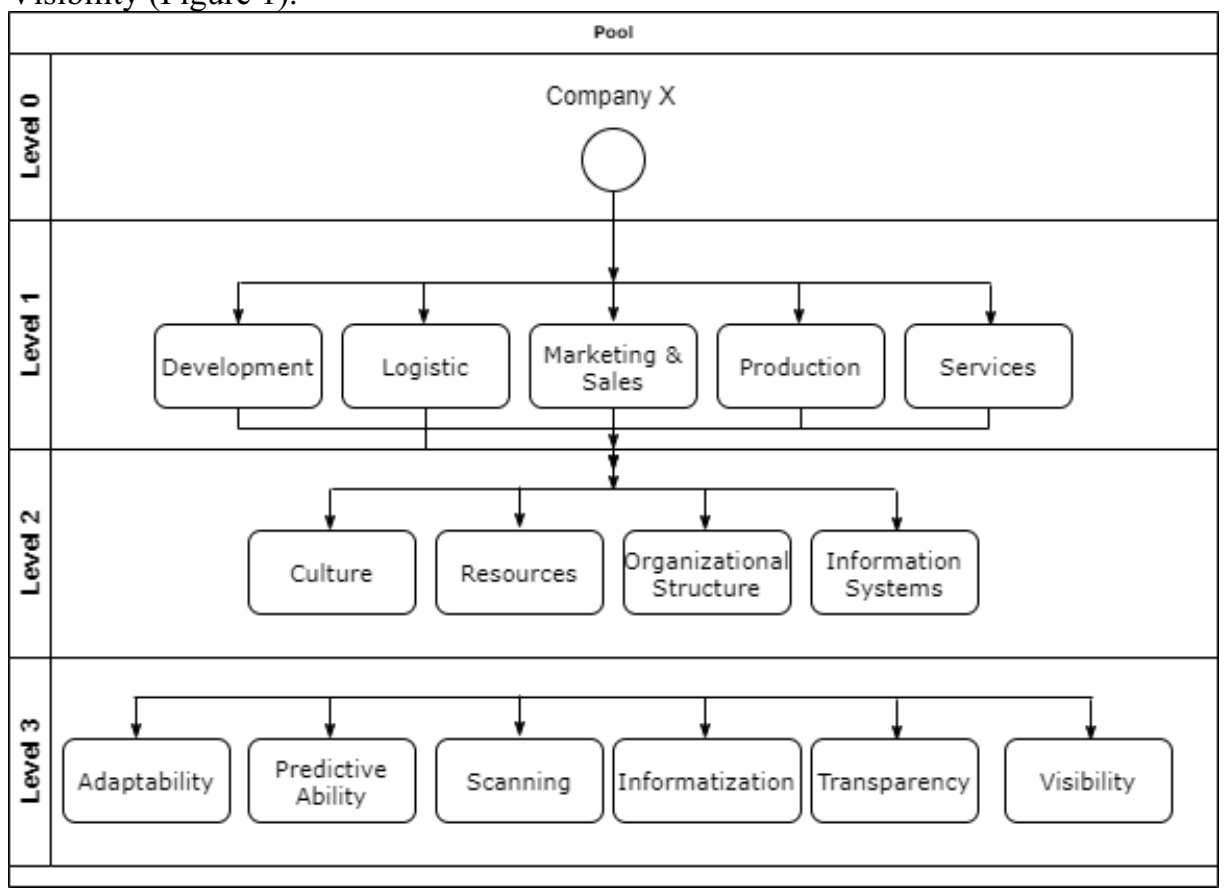

Figure 1. Structure ACATECH, from adapted [6].

This methodology occurs through the elaboration of a questionnaire (script), mapping the questions in all axes so that all levels and sub-levels are included, where people linked to the company and from different areas can answer, thus ensuring that they do not there is a trend or influence on the result, consequently contributing to the mapping and increased maturity [6]. 
The proposed structure is described in Figure 2, which after compiling the responses to the applied form, the next phase is to encompass the AHP. The hierarchical structure is assembled in AHP, allowing that through the responses interpreted by the ACATECH form, the comparisons created by the AHP are filled out. Based on the result obtained, it is possible to simulate scenarios, prioritizing other pillars (Adaptability, Predictive Capacity, Digitization, Computerization, Transparency, Visibility), being able to analyze the impact of changes and future strategies, thus helping decision making.

As mentioned, the ACATECH + AHP structure, proposed by [7], which is a synergy between the ACATECH method and the MCDM, AHP model, brings great detail when interpreting the result provided by the application of the structure
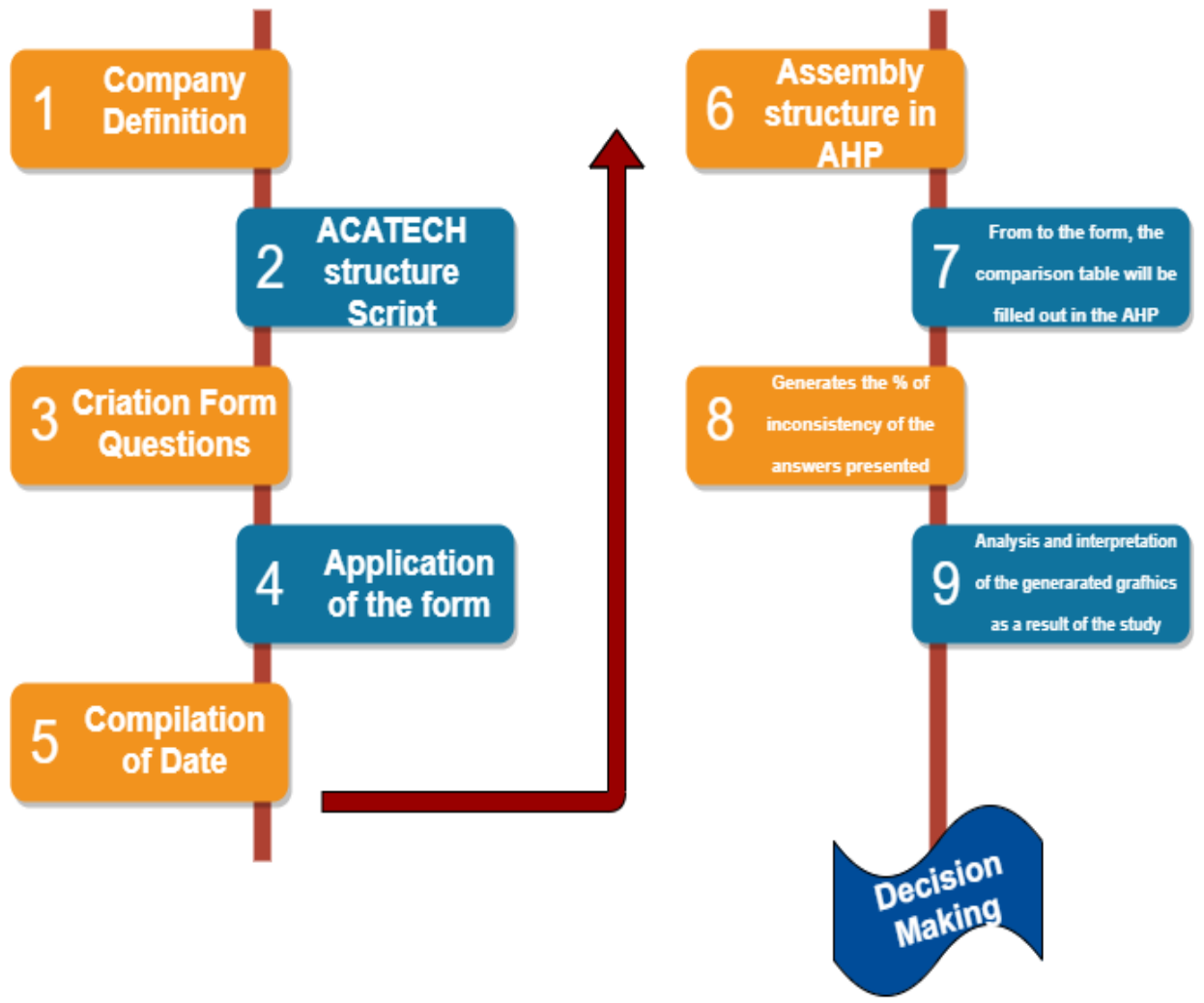

Figure 2. ACATECH + AHP Method Steps, adapted from [7].

\section{Preliminary Discussion}

In [1] the AI4MI framework is applied in a mining company called Master Drilling. Its goal is to be the first point of contact for any customer who wants to expand their current mining operations or start new mining operations. Thus, the objective focuses on how maturity indexing is used to identify the strengths and weaknesses of Master Drilling. The authors needed to change the ACATECH structure to suit the company's reality, together with a readiness measurement model, commissioned by the IMPULS Foundation of the German Engineering Federation. 
In [8], the authors applied the AI4MI framework in 3 cases of several large Danish manufacturing industrial companies, intending to obtain an understanding of how the company is in the relation of the requirements of Industry 4.0, to assess their digital maturity and to guide the definition of a script to address the digital transformation in the companies in question, suggesting improvement activities according to the obtained result. The authors pointed out the need, after application, for a large team of experts to carry out the evaluation process, especially to translate the data collected and mapped into recommendations for improvement, also pointing out the difficulty in interpreting the result provided by ACATECH.

In addition to the difficulty of interpretation, we find it difficult to find work applying ACATECH, or this factor may be correlated with its complexity in interpreting the results.

Thus, we can see that other authors also encountered limitations during the application and when interpreting the results, having the need to adapt the model associated with another one or to outsource the interpretation to a more technical group.

In the same way as the authors mentioned above, the author [7] commented on the challenges, limitations found during the application of the ACATECH tool, which motivated to associate decision-making with an aid method, in a way to interpret and instruct the analyst as to the result obtained, which occurs through statistical, graphical interpretations, simulating scenarios (Figure 3), more playfully and intuitively, when compared to the product supplied to ACATECH, a radar graph. However, when analyzing the ACATECH + AHP model, which proved to be very advantageous, the limitation due to the extension of the method was observed, becoming tiring, and also during the completion of the AHP strike, it can generate confusion.

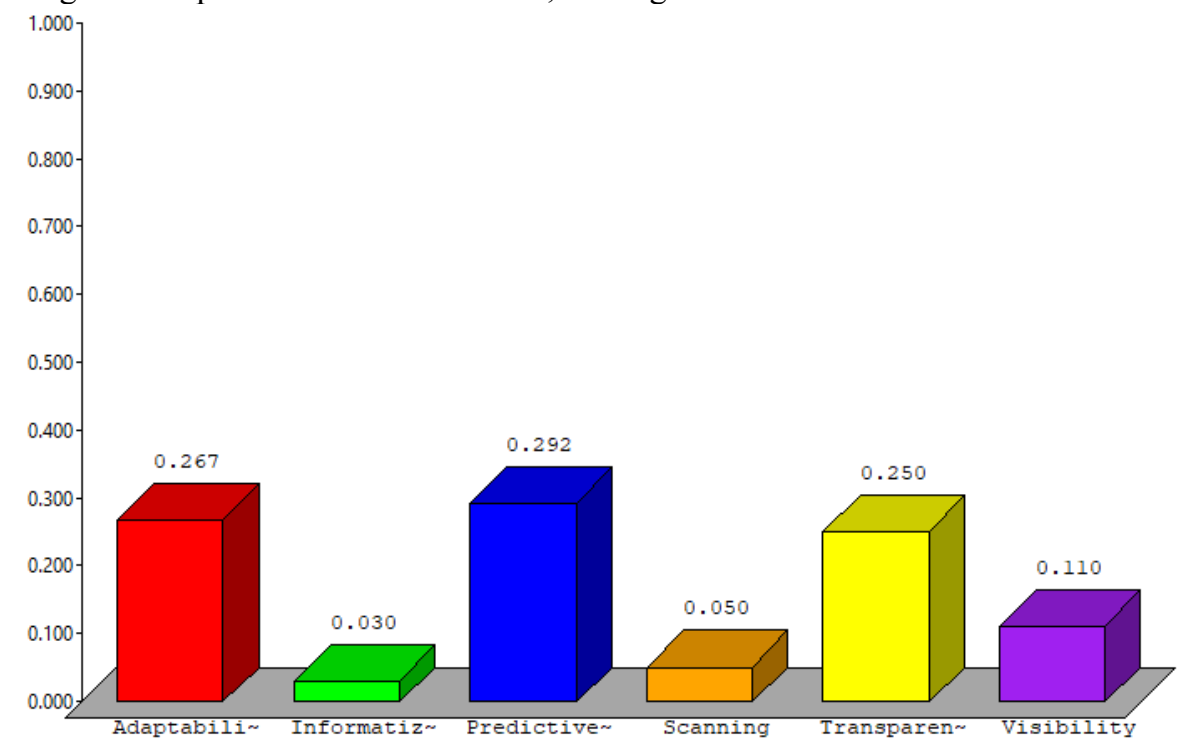

Figure 3. ACATECH + AHP Method Steps, taken from [7].

Figure 3 reveals how the ACATECH + AHP structure presents the interpretation of the maturity index measurement result. 
After performance measurement, actions must be established to optimize the points to be developed or the points that impact according to the established strategy.

Figure 4 provides the following explanation: Organizations must inform/implement their existing strategy, linking it as data entry, with the technological developments that Industry 4.0 makes available (IoT, Big Data, Cloud). Another entry, which is on the rise, is industries or the processing of products or services linked to sustainability, not just products scheduled to be reused or recyclable, with return, but more mature and sustainable.

Thus, the organizational strategy must reflect three new dimensions, namely, the creative use of technology, unlocking innovation through collaboration and co-creation, as well as a sustainability agenda to create a competitive advantage. In this way, the organization would implement its strategy using a performance measurement framework, with behavior and measurement in a social environment. Finally, organizations must manage their performance not only by measuring internally, but also in collaborative networks and social media and, in some way, by measuring social factors, requirements, customer, and competitor acceptability. The result of this stage is the evolution of collaborative networks and social evolution [9].

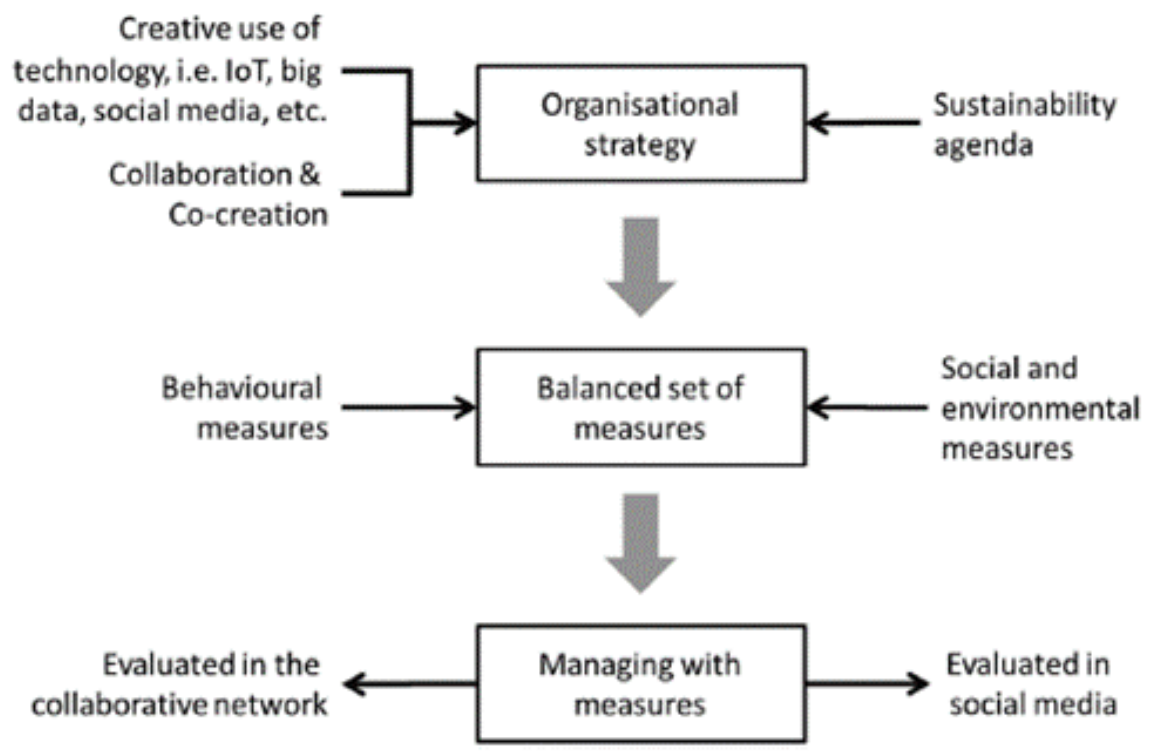

Figure 4. ACATECH + AHP Method Steps, taken from [1].

\section{Conclusion}

Through this article, we were able to demonstrate and complete the displayed model ACATECH + AHP, when compared with the ACATECH structure itself and other methods formed from it, shown to be very efficient, practical, effective and quick to assess the maturity index. The main conclusion is that ACATECH is a great tool, but in all cases analyzed, it shows confusion in the relationship with the interpretation of the 
result, being necessary, as reported in the mentioned article, an outsourcing of the interpretation of the results, so that the measures and action plans are taken correctly.

Research shows that resources widely grouped into the categories of processes, people, systems, and culture allow the organization to deal with the changing environment and modify its performance system according to its changes and evolution.

For performance to effectively contribute to the management of the organization in question, it is necessary to provide feedback loops on the measures, that is, periodically monitor their evolution, problems, and success. This leads to the recognition of various types of performance measures, especially for long-term business improvement initiatives.

It is worth mentioning that old performance measures must be excluded after the evolved system, so that they give space to measures that reflect the reality of the scenario and the current need.

As next work, implement the ACATECH + AHP structure in cases of companies with operations in different markets, not only to measure maturity in different segments, but mainly to measure the difficulty of implementing in several sequences, as well as correlating the strengths and between companies and between companies in similar markets.

\section{Acknowledgment}

The authors would like to thank the Pontifical Catholic University of Parana (PUCPR) and Robert Bosch CtP for the financial support to the development of this research.

\section{References}

[1] G.J. Maasz and H. Darwish, Towards an Initiative-Based Industry 4.0 Maturity Improvement Process: Master Drilling As a Case Study, South African Journal of Industrial Engineering, 2018, Vol. 29(3), pp. 92-107. doi: 10.7166/29-3-2052.

[2] J. Basl, Pilot Study of Readiness of Czech Companies to Implement the Principles of Industry 4.0, Management and Production Engineering Review, 2017, Vol. 8(2), pp. 3-8. doi: 10.1515/mper-20170012

[3] M. Kennerley and A. Neely, Measuring performance in a changing business environment, International Journal of Operations and Production Management, 2003, Vol. 23(2), pp. 213-229. doi: $10.1108 / 01443570310458465$.

[4] K. Hua Tan, K. Platts and J. Noble, Building performance through in-process measurement: Toward an "indicative" scorecard for business excellence, International Journal of Productivity and Performance Management, 2004, Vol. 53(3), pp. 233-244. doi: 10.1108/17410400410523774.

[5] H. Fatorachian and H. Kazemi, A critical investigation of Industry 4.0 in manufacturing: theoretical operationalization framework, Production Planning and Control, 2018, Vol. 29(8), pp. 633-644. doi: 10.1080/09537287.2018.1424960.

[6] R. Anderl, Industrie 4.0 Maturity Index, Acatech, 2017, doi: ISSN 2192-6174.

[7] T. Rocha, E. Loures and A. Szejka, Evaluation of the Industry 4.0 Acatech Maturity Index in the metallurgical sector based on the AHP method, 2020, in press.

[8] M. Colli, U. Berger, M. Bockholt et al. A maturity assessment approach for conceiving context-specific roadmaps in the Industry 4.0 era, Annual Reviews in Control, 2019, pp. 1-13. doi.org/10.1016/j.arcontrol.2019.06.001.

[9] S.S. Nudurupati, S. Tebboune and J. Hardman, Contemporary performance measurement and management (PMM) in digital economies, Production Planning \& Control, 2016, 27:3, pp. 226-235, doi: $10.1080 / 09537287.2015 .1092611$ 
[10] M. Artz, C. Homburg and T. Rajab, Performance-measurement system design and functional strategic decision influence: The role of performance-measure properties, Accounting, Organizations and Society, 2012, Vol. 37(7), pp. 445-460. doi: 10.1016/j.aos.2012.07.001

[11] F. Ansari, et al. A problem-solving ontology for human-centered cyber physical production systems, CIRP Journal of Manufacturing Science and Technology, 2018, Vol. 22, pp. 91-106. doi: 10.1016/j.cirpj.2018.06.002

[12] J. Basl, 'Companies on the Way to Industry 4.0 and their Readiness, Journal of Systems Integration, 2018, Vol. 9(3), pp. 3-6. doi: 10.20470/jsi.v9i3.351.

[13] M.S. Garcia-Cascales and M.T. Lamata, Multi-criteria analysis for a maintenance management problem in an engine factory: Rational choice, Journal of Intelligent Manufacturing, 2011, Vol. 22(5), pp. 779788, doi: 10.1007/s10845-009-0290-x.

[14] B. Gärtner, Step-by-step to Industrie 4.0, Plant Engineering, 2018, Vol. 72(1), pp. 14-15.

[15] T.D.H. Gusberti and M.E.S. Echeveste, An Organizational Capability-Based Performance Measurement Model for Technology Conversion Process, Journal of CENTRUM Cathedra: The Business and Economics Research Journal, 2012, pp. 225-242. doi: 10.7835/jcc-berj-2012-0076.

[16] M. Hudnurkar et al., Development of a balanced scorecard-based supplier collaborative performance index, International Journal of Productivity and Performance Management, 2018, Vol. 67(2), pp. 401425. doi: 10.1108/IJPPM-05-2016-0084.

[17] S. Mittal et al., A critical review of smart manufacturing \& Industry 4.0 maturity models: Implications for small and medium-sized enterprises (SMEs), Journal of Manufacturing Systems, 2018, Vol. 49(June), pp. 194-214, doi: 10.1016/j.jmsy.2018.10.005.

[18] S.K. Rao and R. Prasad, Impact of 5G Technologies on Industry 4.0, Wireless Personal Communications, 2018, Vol. 100(1), pp. 145-159. doi: 10.1007/s11277-018-5615-7. 